\title{
Technè
}

La science au service de l'histoire de l'art et de la préservation des biens culturels

$42 \mid 2015$

Science et conservation

\section{Les Trois baigneuses de Courbet (musée du Petit Palais) : restauration et découvertes}

Courbet's Three Bathers (Musée du Petit Palais): restoration and findings

\section{Isabelle Collet et Claudia Sindaco-Domas}

\section{(2) OpenEdition}

Journals

Édition électronique

URL : http://journals.openedition.org/techne/7176

DOI : 10.4000/techne.7176

ISSN : 2534-5168

Éditeur

C2RMF

Édition imprimée

Date de publication : 1 décembre 2015

Pagination : 103-108

ISBN : 978-2-7118-6249-8

ISSN : $1254-7867$

Référence électronique

Isabelle Collet et Claudia Sindaco-Domas, «Les Trois baigneuses de Courbet (musée du Petit Palais) : restauration et découvertes », Technè [En ligne], 42 | 2015, mis en ligne le 01 décembre 2015, consulté le 11 mars 2021. URL : http://journals.openedition.org/techne/7176 ; DOI : https://doi.org/10.4000/ techne. 7176

\section{(c) (i) (2) $\Theta$}

La revue Technè. La science au service de l'histoire de l'art et de la préservation des biens culturels est mise à disposition selon les termes de la Licence Creative Commons Attribution - Pas d'Utilisation Commerciale - Pas de Modification 4.0 International. 


\section{Isabelle Collet Claudia Sindaco-Domas \\ Les Trois baigneuses de Courbet (musée du Petit Palais) : restauration et découvertes}

Courbet's Three Bathers (Musée du Petit Palais): restoration and findings

Résumé. Courbet a peint les Trois baigneuses vers 1865-1868, sans pouvoir achever sa composition après son départ en exil en Suisse. L'œuvre, restée à Paris, est entrée dans les collections du Petit Palais en 1909 grâce à une donation de Juliette Courbet. Ce tableau présente la particularité d'être peint à l'huile sur papier et d'avoir été agrandi en cours d'exécution. Restauré en 1973, puis marouflé sur une nouvelle toile en 1977, il a fait de nouveau l'objet d'une intervention en 2014. Ce travail sur la couche picturale a été précédé d'une étude au C2RMF qui a permis de mieux comprendre la genèse de l'œuvre et son évolution : d'une figure d'odalisque présentée sur un format horizontal, elle est devenue une composition à trois personnages, en format vertical. La restauration de la couche picturale menée en 2014 au Petit Palais a été l'occasion d'examiner de plus près la technique picturale de Courbet, de la confronter à d'autres peintures de l'artiste et d'en restituer la qualité intrinsèque en respectant son caractère inachevé.

Mots-clés. Gustave Courbet, Trois baigneuses, technique picturale, cuvre inachevée, repeints, allègement.

\begin{abstract}
Courbet painted the Three Bathers in 1865-68, but was unable to finish his composition before moving to Switzerland to live in exile. The painting remained in Paris and was donated by Juliette Courbet to the Petit Palais in 1909. What is distinctive about this work is that it was painted on paper in oil paint and was enlarged during its execution. Restored in 1973, then remounted on new canvas in 1977, it underwent another restoration in 2014. This work on the pictorial layers was preceded by a study conducted at the C2RMF that provided information about the genesis and development of the painting: what started out as a figure of an odalisque in a horizontal format turned into a three-figure composition in a vertical format. The restoration of the pictorial layers completed in 2014 at the Petit Palais furnished an opportunity to examine Courbet's painting technique more closely, to compare it to other paintings by the artist and to render its intrinsic quality by respecting its unfinished character.

Keywords. Gustave Courbet, Three Bathers, pictorial technique, unfinished work, repaints, lightening.
\end{abstract}

Le Petit Palais, musée des beaux-arts de la Ville de Paris, conserve un ensemble remarquable d'œuvres de Gustave Courbet. Le peintre fut l'un des plus célèbres proscrits de la Commune. Après son décès survenu en 1877 à la Tour-dePeilz, sa plus jeune sœur, Juliette Courbet (1831-1915), a veillé à la réhabilitation de l'artiste et à la pérennité de son œuvre. Dès 1881, la Ville de Paris avait fait l'acquisition d'une grande toile, La Sieste pendant la saison des foins (1867), lors d'une vente aux enchères. En 1900, le grand portrait hommage intitulé Pierre-Joseph Proudhon et ses enfants (1865) est à son tour acheté par la Ville. Juliette Courbet a servi d'intermédiaire pour la donation en 1906 d'une ouvre majeure, Les Demoiselles des bords de Seine (1856), complétée par la donation de plusieurs portraits de famille dont l'Autoportrait au chien noir (1844). Ces peintures sont rassemblées dans une salle Courbet inaugurée en 1909, au rez-de-jardin. Le tableau Trois baigneuses y figure alors en bonne place. L'œuvre est citée dans le catalogue des collections rédigé par Henry Lapauze en 1910 : «Les Trois baigneuses s'égalent à La Source (fig. 7), qui est restée chez Melle Juliette Courbet. Elles datent de 1868. Elles ont la même saveur que les meilleurs nus réalistes de Courbet : la jeune femme qui forme le centre du tableau, et qui va descendre dans l'eau, est un des beaux morceaux de la fin de sa carrière ; la chair des trois baigneuses est palpitante de vie ${ }^{1}$ ». Les nus féminins ont très tôt fait la réputation du peintre qui aurait pu, sans difficulté, vendre une œuvre aussi séduisante. Il n'en a rien été car Courbet n'a pas eu le temps d'achever cette composition.

Peinte peu de temps avant le départ forcé de Courbet pour la Suisse, l'œuvre a connu un destin mouvementé. Elle est volée chez l'artiste passage du Saumon et proposée à la vente chez un encadreur où elle est retrouvée en 1874 et restituée à l'artiste ${ }^{2}$. À cette date, Étienne Baudry et Jules Castagnary constatent que «cette grande toile paraît inachevée ». Restée dès lors dans l'atelier parisien, elle ne figure ni dans la vente après décès du 26 novembre 1877, ni à l'exposition 
Courbet organisée à l'École des beaux-arts en 1882. Son arrivée au Petit Palais en 1909 est donc sa première présentation officielle au public. L'ouvre qui mesure 126 x $96 \mathrm{~cm}$ est alors dotée d'un beau cadre mouluré et doré à la feuille, ce qui confère au tableau le statut d'une œuvre achevée.

\section{Une carrière internationale}

L'exposition Courbet organisée en 1929 au Petit Palais marque le début d'une reconnaissance historique du peintre d'Ornans décédé cinquante-deux ans auparavant. Notre tableau figure sous le numéro 54 du catalogue, avec le titre : Les Baigneuses ${ }^{3}$. Dès lors, les expositions Courbet se multipliant de par le monde, le tableau va connaître une carrière internationale, dont un passage remarqué à la Biennale de Venise en 1954. Cette étape nous intéresse particulièrement du point de vue de l'évolution de l'état de l'œuvre, car le tableau est reproduit en couleurs dans le catalogue Courbet alla XXVII biennale di Venezia.

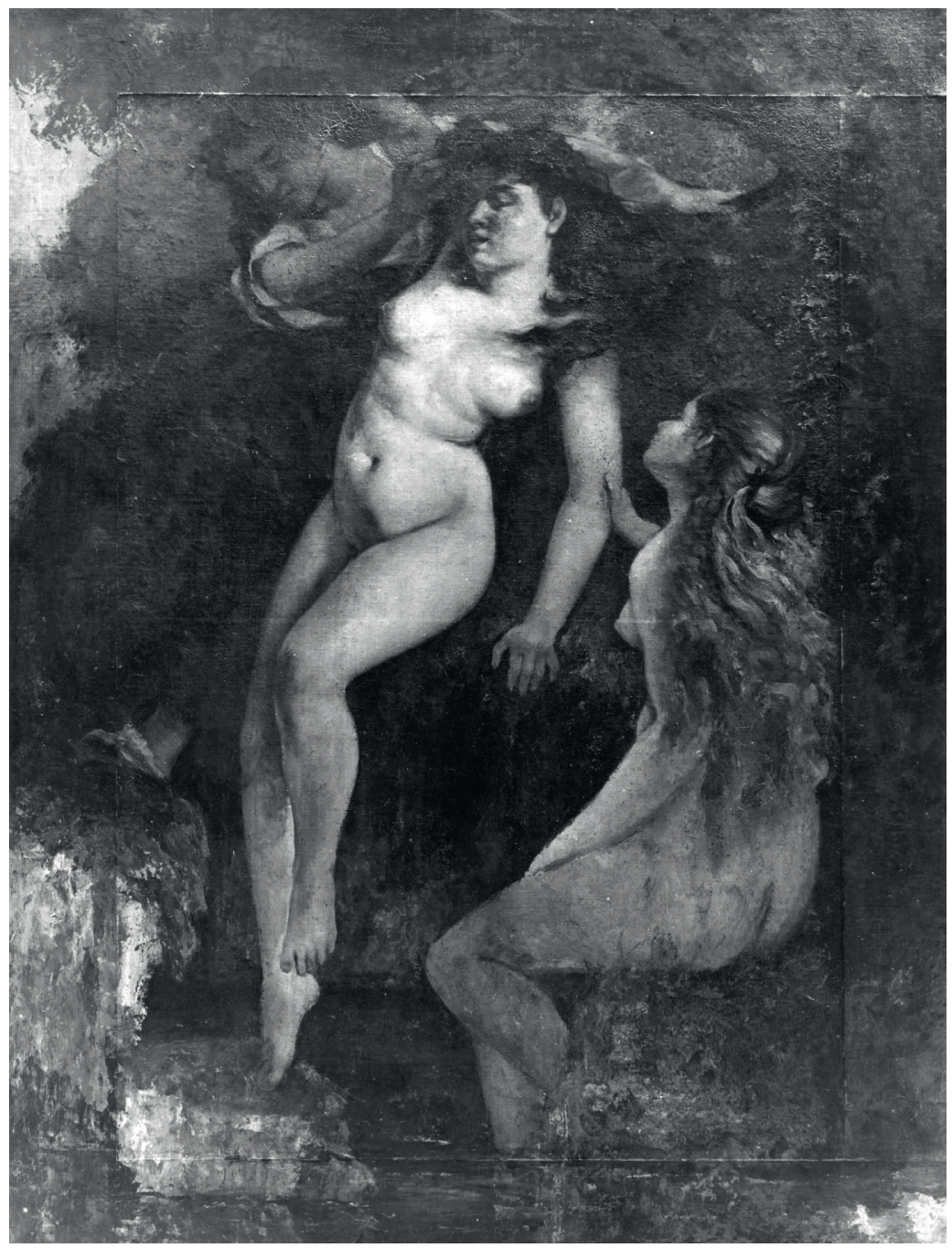

Un tirage noir et blanc de l'agence Bulloz conservé au Petit Palais est daté au revers du $1^{\text {er }}$ trimestre 1970 (fig. 1). Ce cliché permet d'établir un constat de l'état de l'œuvre avant sa restauration de 1973 : une première restauration est confiée à Jacques Roullet, chef de l'atelier de restauration des peintures du musée du Louvre demeurant 39 boulevard Saint-Jacques. La facture datée du 7 décembre 1973, seul document conservé dans le dossier, indique : " Nettoyage, enlèvement des taches et régénération du vernis ${ }^{4}$. » Il n'est donc pas fait mention du support dans cette opération facturée 300 francs. Un mémoire de restauration adressé au musée le 6 décembre 1976 facture un « rentoilage » pour un montant de 4000 francs par l'atelier Rostain ${ }^{5}$. L'œuvre est absente de la rétrospective Courbet présentée au Grand Palais puis à la Royal Academy de Londres, du 30 septembre 1977 au 19 mars 1978. En octobre 1977, notre tableau s'envole pour New Delhi à destination d'une exposition intitulée Modern French painting. À son retour en février 1978, il est constaté que le « rentoilage est raté : une bosse était visible le jour même du retour du tableau au musée ${ }^{6}{ }$. Le marouflage est refait par l'atelier Rostain en avril 1978. L'œuvre va pouvoir repartir à Hambourg après une légère intervention de William Langelaan (restaurateur des musées nationaux) qui constate qu'il " faudrait faire une restauration plus poussée $^{7}$ ».

Le tableau part de nouveau en prêt à l'exposition Courbet und Deutschland ${ }^{8}$. Les Baigneuses sont reproduites en couleurs sur la couverture du catalogue (cliché RMN). Le cliché de médiocre qualité est malheureusement difficile à interpréter ${ }^{9}$. Le vernis présente de larges plages jaunâtres, et l'on retrouve sur la joue gauche les tâches disgracieuses déjà constatées en 1970. L'œuvre est reproduite à plusieurs reprises dans des catalogues d'exposition durant les années 1980 et 1990. Puis une nouvelle prise de vue

Fig. 1. Trois baigneuses. Tirage photographique noir et blanc. Le vernis encore transparent laisse bien visible le fond arboré de la composition; la joue gauche de la baigneuse brune est déjà marquée de taches brunes ainsi que le sein gauche, en revanche son corps ne présente pas de marques brunes prononcées, mais cela peut être dû à la mauvaise qualité du tirage ; les zones de raccord entre la feuille centrale et les quatre bandes ajoutées en périphérie sont bien visibles sur l'image, ce qui indique probablement des problèmes d'adhérence entre la toile de doublage et le bord des feuilles de papier épais dès cette date. (C) Photo Bulloz, vers 1970, archives du Petit Palais. 
en couleurs est effectuée en 1996 par l'agence photographique des musées de la Ville de Paris. Les repeints désaccordés sont désormais bien visibles sur le corps de la baigneuse brune.

\section{La restauration de 2014 : nouveaux enjeux}

Lorsque la fondation Beyeler demande le tableau en prêt, le musée charge Jean-François Hulot et Cécile des Cloizeaux de faire un constat détaillé afin de vérifier si l'œuvre peut voyager. Les restaurateurs rendent leurs conclusions en novembre 2013. Le marouflage opéré en 1978 par l'atelier Rostain reste suffisamment stable pour permettre à l'œuvre de voyager. En revanche, l'état de surface est peu satisfaisant compte tenu de l'oxydation du vernis et de l'assombrissement des nombreux repeints. Le prêt étant accordé pour l'exposition de Bâle en 2014, le Petit Palais décide de confier la restauration de l'œuvre à Claudia Sindaco. L'opération bénéficie du soutien financier de la fondation Beyeler. Il est toutefois prévu de n'entreprendre cette restauration qu'après une analyse détaillée de la peinture afin de mieux comprendre la genèse de ce tableau inachevé et complexe.

L'étude menée par le C2RMF sous la direction de Bruno Mottin a fourni des réponses essentielles à la compréhension de l'œuvre (voir article de Bruno Mottin page 101). Elle confirme l'hypothèse d'un agrandissement du format par Courbet lui-même et démontre qu'une première composition avait été ébauchée sur la feuille centrale travaillée en format horizontal.

La restauration de 2014 concerne exclusivement la couche picturale. Elle doit permettre de purger l'œuvre des repeints désaccordés qui masquent sans doute d'anciens accidents de surface (hypothèse infirmée après l'allègement). L'enjeu de cette intervention sera donc d'approcher l'état dans lequel Courbet a laissé ces Baigneuses en quittant son atelier parisien. Cette recherche d'un état originel doit néanmoins prendre en compte l'évolution de la matière qui a rendu davantage visibles, par une transparence accrue, les étapes antérieures.

\section{La technique picturale de Courbet, observations et documents}

L'abondante correspondance de Courbet et les témoignages de ses contemporains ${ }^{10}$ nous renseignent sur les méthodes de travail de l'artiste, les matériaux qu'il aimait employer et ses recherches plastiques. Avant d'entreprendre la restauration, nous avons confronté nos observations étayées par l'étude du C2RMF avec ces sources historiques.

L'œuvre a été réalisée à la fin de la carrière du peintre, dans les années 1865-1868. Courbet peint alors depuis une quinzaine d'années avec constance et rapidité, en employant une technique puissante et personnelle. Les descriptions de ses amis montrent Courbet à l'œuvre dans son atelier « arrêté tour à tour devant chaque chevalet, grattant par ici, retouchant par-là, n'attaquant que rarement une toile vierge ${ }^{11} »$. D'autres récits soulignent sa vitesse et sa sûreté de travail, comme Max Claudet qui l'a vu travailler «à peine deux heures pour couvrir une toile d'un mètre ${ }^{12}$ ! ».

Le support employé, un papier, est assez peu utilisé par l'artiste et plutôt réservé à des esquisses préparatoires ou à des œuvres de petit format. L'examen rapproché révèle qu'il est d'une tonalité brune, sans préparation. Le seul témoignage sur ce support est donné par Alexandre Shanne qui mentionne avoir vu Courbet dans ses années de formation peindre des grands formats avec «du papier gris épais, préparé à l'huile et tendu sur des châssis ${ }^{13}$ ».

Le support est composé de cinq feuilles assemblées à joints vifs. Le peintre a modifié sa composition en agrandissant le format originel par l'adjonction de quatre bandes de papier (voir article de Bruno Mottin page 101). Pour maintenir ces agrandissements, il a fait pratiquer un marouflage. Paris comptait, à la fin du XIX ${ }^{\mathrm{e}}$ siècle, de nombreux ateliers qui pratiquaient des rentoilages ou marouflages à la demande d'artistes ou de particuliers ${ }^{14}$. Émile Rostain, restaurateur de support toile, est le premier à examiner le revers en 1976 pour reprendre les décollements du marouflage original. Il constate alors que le renfort du papier par une toile «a été réalisé au moment où Courbet a agrandi le tableau ${ }^{15}$ ».

La première composition centrale est proche d'un format grand aigle. Les reprises constatées à la radiographie sont fréquentes chez l'artiste et souvent relatées par ses proches ou par Courbet lui-même. Parmi ces écrits, nous en citerons deux, celui de Silvestre : «Il dessine grosso modo les personnages, les construit, les reconstruit jusqu'à trois fois de pied en cap ${ }^{16}$ ", celui de Courbet : "J'ai repris mon Retour de foire auquel il manquait bien des choses et dans lequel il y avait un défaut de perspective, puis je l'ai agrandi d'un quart ${ }^{17}$. »

La réflectographie n'a révélé que des traces de fusain pour mettre en place les formes principales. Courbet peint généralement après avoir sommairement indiqué les lignes directrices de sa composition. Il est rare, s'agissant de petits formats, qu'il réalise un croquis ou un dessin à part. Plusieurs amis évoquent un dessin à la craie blanche sur une préparation sombre ${ }^{18}$, qu'il modifie souvent en cours de travail.

Dans les Trois baigneuses, la teinte brune du papier sert de ton de fond à l'œuvre. Courbet affectionnait les préparations sombres qui lui permettaient de travailler du foncé vers le clair, par éclaircissement progressif. Il s'en explique à un élève au cours d'une leçon : "Cherche une teinte plus foncée que celle-là et plaque cette teinte avec ton couteau, ensuite attaque par gradations les nuances les moins intenses, enfin tu n'auras qu'à faire luire les clairs ${ }^{19}$. » Cette méthode lui permettait de mettre en valeur les modelés et de mieux faire ressortir les lumières.

Le caractère inachevé de l'œuvre est précieux pour voir la facture de Courbet et comprendre ses étapes de travail. Les témoignages parlent souvent d'une matière très compacte au départ, appliquée au couteau et à la brosse large, au chiffon ou au doigt, puis progressivement adoucie par des touches de plus en plus fluides et colorées. Les effets de matière sont donc variés. Certaines zones d'un tableau peuvent recevoir 
des couches successives et d'autres restent à peine esquissées. Des amis évoquent pour sa matière des tableaux d'anciens maîtres, d'autres des empâtements semblables à un «crépi ${ }^{20}$ ».

Dans le tableau du Petit Palais, le personnage central peint avec un modelé subtil contraste avec la figure de la jeune femme blonde, rapidement ébauchée et jamais terminée. Pour la végétation, Courbet juxtapose des petites touches ou applique des glacis dans le frais pour animer la surface. Pour la jeune fille blonde, la matière conserve l'empreinte des brosses larges et du couteau à peindre. Dans cette zone, nous percevons par transparence des feuillages sombres, témoin de la première composition.

Concernant sa palette, les amis du peintre évoquent l'emploi de couleurs ordinaires conservées dans des vessies. composée de blanc de plomb, de vermillon, d'ocre jaune et de noir. Des témoignages relatent qu'au moment de peindre, « après avoir regardé avec soin son modèle il combinait les tons, il en préparait trois fondamentaux pour la lumière, la demi-teinte et l'ombre, puis il disposait les couleurs franches sur le haut de sa palette ${ }^{21} »$. Les carnations des trois baigneuses ont été peintes en respectant cette méthode de travail.

Dans la correspondance de Courbet, nous n'avons trouvé aucune mention de vernis final ou intermédiaire appliqué par l'artiste lui-même. Un témoignage de Fleury raconte l'avoir vu mouiller à l'éponge des toiles en cours d'exécution pour les présenter à ses visiteurs ${ }^{22}$. D'autres lettres de Courbet indiquent qu'il laissait le soin à ses marchands de vernir et d'encadrer ses œuvres, comme il était d'usage de le faire à la fin du XIX ${ }^{\mathrm{e}}$ siècle $^{23}$. Le tableau des Trois baigneuses, inachevé et non exposé du vivant de l'artiste, n'a certainement pas été verni. Il est probable que Juliette Courbet, au moment de la donation, ait fait vernir et encadrer la peinture. Ce vernis a

été nettoyé puis régénéré lors des restaurations de 1973 et 1978. Ces couches de vernis sont composées de résine naturelle comme l'indique leur fluorescence à l'ultra-violet.

\section{La restauration de 2014}

Les interventions esthétiques, faites pour améliorer l'état de présentation de l'œuvre, se sont déroulées au musée du Petit Palais et ont été suivies à chaque étape par le conservateur responsable des collections, Isabelle Collet. La technique peu conventionnelle de l'artiste, les nombreuses reprises et repentirs, laissaient craindre une matière fragile et un allègement délicat. Les vernis de restauration épais et oxydés avaient perdu leur transparence. Ils gênaient l'appréciation des plans et des contrastes, et les larges repeints altérés dans le personnage central troublaient la compréhension du modelé (fig. 2 et 3).

Nous avons opté pour un travail prudent et progressif, conforme à la méthodologie définie par Wolbers et Cremonesi pour les opérations de décrassage et d'allègement du vernis. Après un décrassage qui a débarrassé la matière d'une couche grise, des petits tests de solubilité ont permis de sélectionner des mélanges de solvants pour amincir les vernis ${ }^{24}$. Un degré d'allègement prononcé, qui laisse une fine couche de vernis ancien, a été choisi à l'issue de ces tests (fig. 5). Les repeints ont été totalement retirés.

Après l'allègement, nous avons réexaminé l'état de présentation. Le tableau a gagné en netteté et en profondeur, le modelé du personnage central a retrouvé ses nuances subtiles. À ce stade du travail, quelques altérations troublent encore la lecture : des usures et des petites lacunes (très anciennes) sont toujours visibles dans le visage du personnage central.

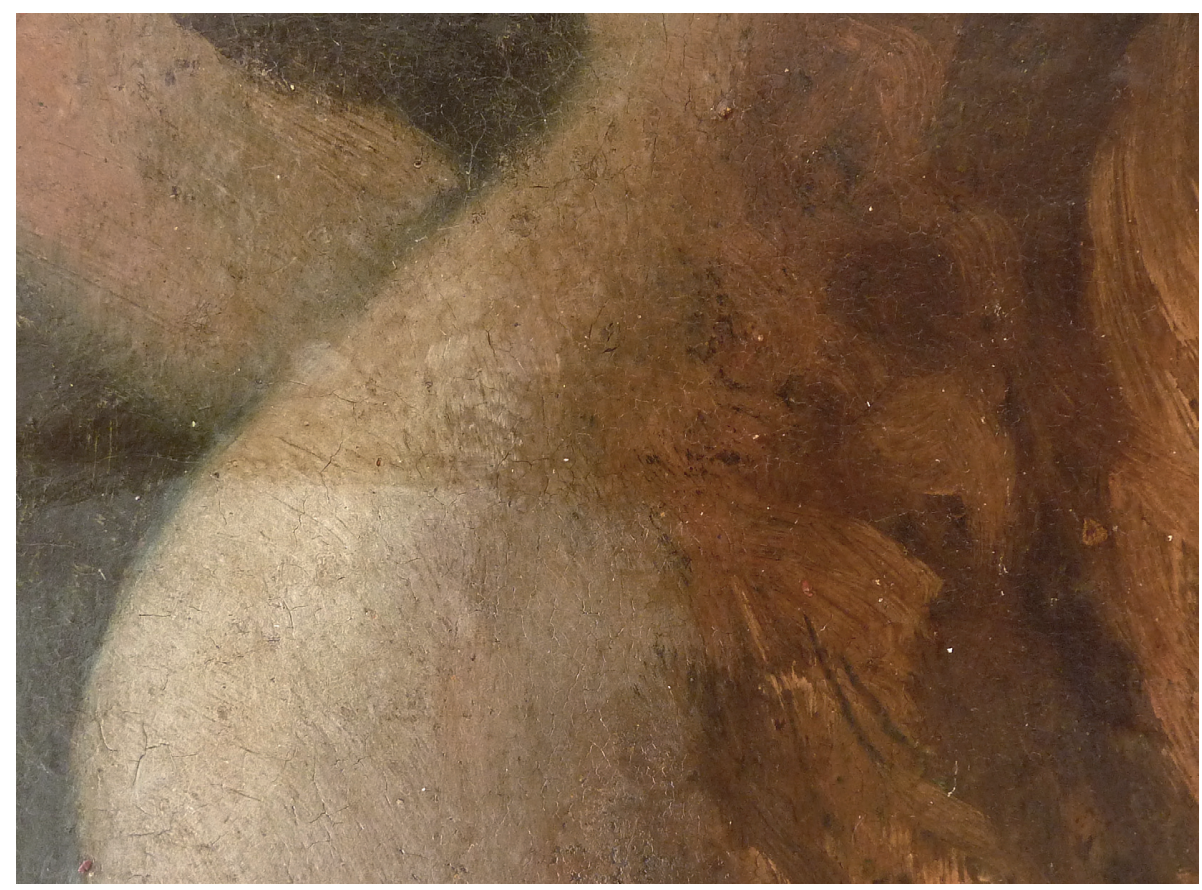

Fig. 2. Détail d'une zone en cours d'allègement. (c) Claudia Sindaco. 


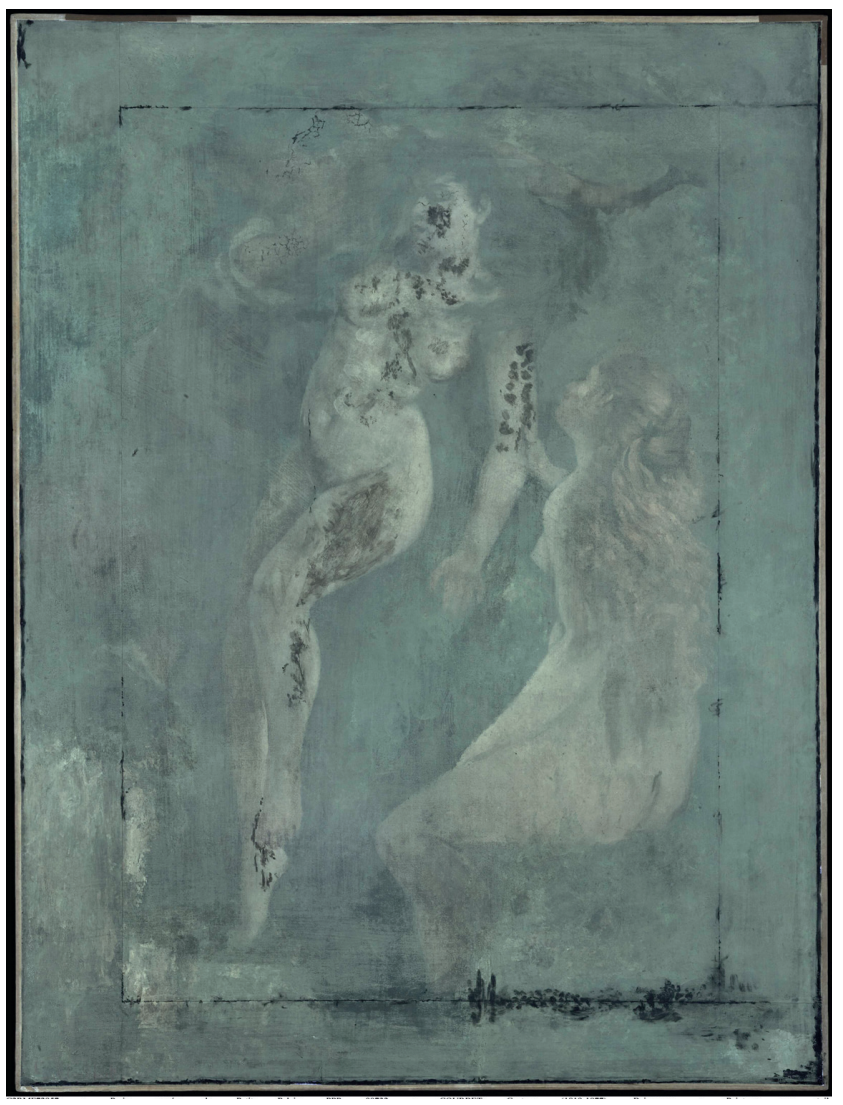

Fig. 3. Photographie sous ultraviolet. Les repeints apparaissent en sombre. (C) C2RMF/Jean-Louis Bellec.

Elles sont alors retouchées pour retrouver le modelé. Les larges craquelures prématurées dans le personnage de l'arrière-plan ont été atténuées afin de redonner plus de netteté aux formes.

Dans le personnage blond, la transparence accrue de l'huile rendait plus perceptibles les sous-couches sombres de la composition initiale ${ }^{25}$. Courbet a laissé cette figure à l'état d'ébauche contrairement à la baigneuse centrale. Le travail de retouche a tenu compte de cette évolution naturelle tout en restant minimal. Nous avons atténué par des glacis certaines zones où les sous-couches de la végétation, trop présentes, brouillaient la lecture. Enfin, nous avons appliqué une fine couche de vernis satiné qui redonne transparence et profondeur à la matière tout en préservant le caractère inachevé de l'œuvre 26 (fig. 6).

\section{Conclusion}

Le tableau des Trois baigneuses restait l'une des œuvres les plus mystérieuses du fonds Courbet conservé au Petit Palais. Les recherches menées en 2014 à l'occasion de son prêt à la fondation Beyeler ont permis de réunir nombre d'informations inédites sur l'histoire matérielle de cette composition à ses différents stades d'élaboration. Euvre muséale, magnifiée

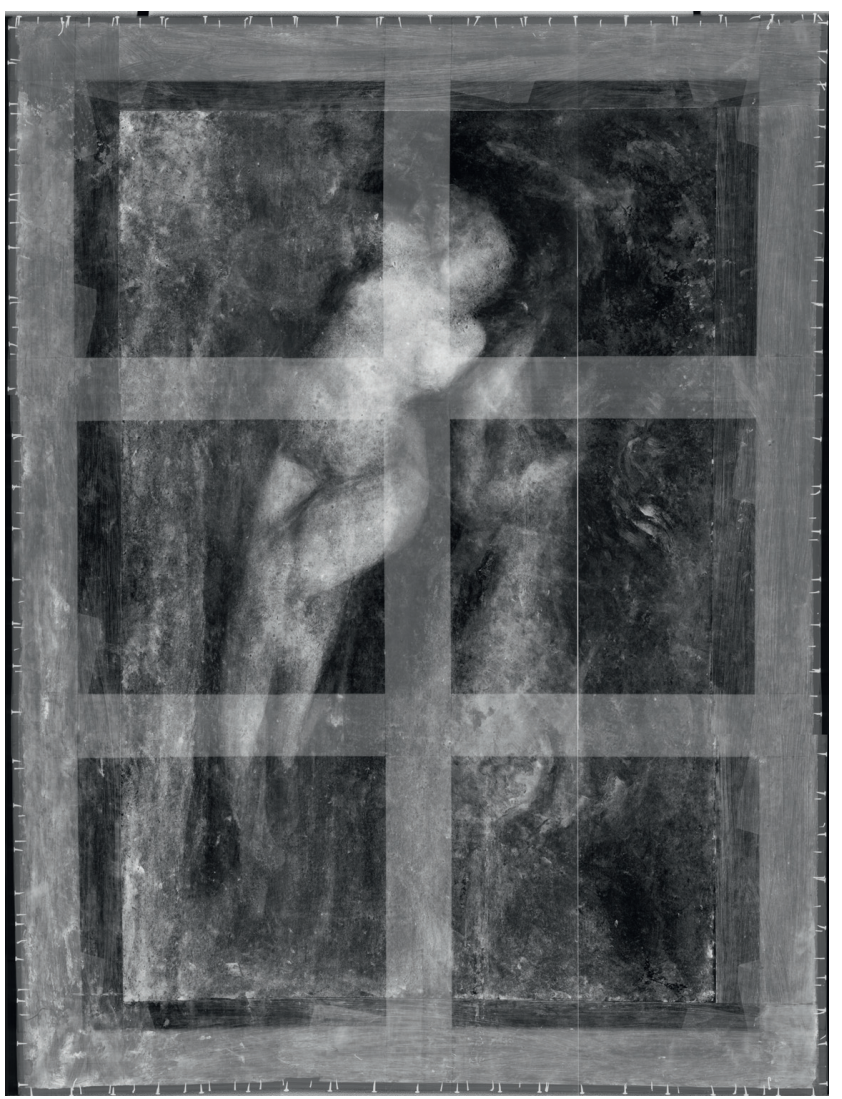

Fig. 4. Radiographie. ( ) C2RMF.

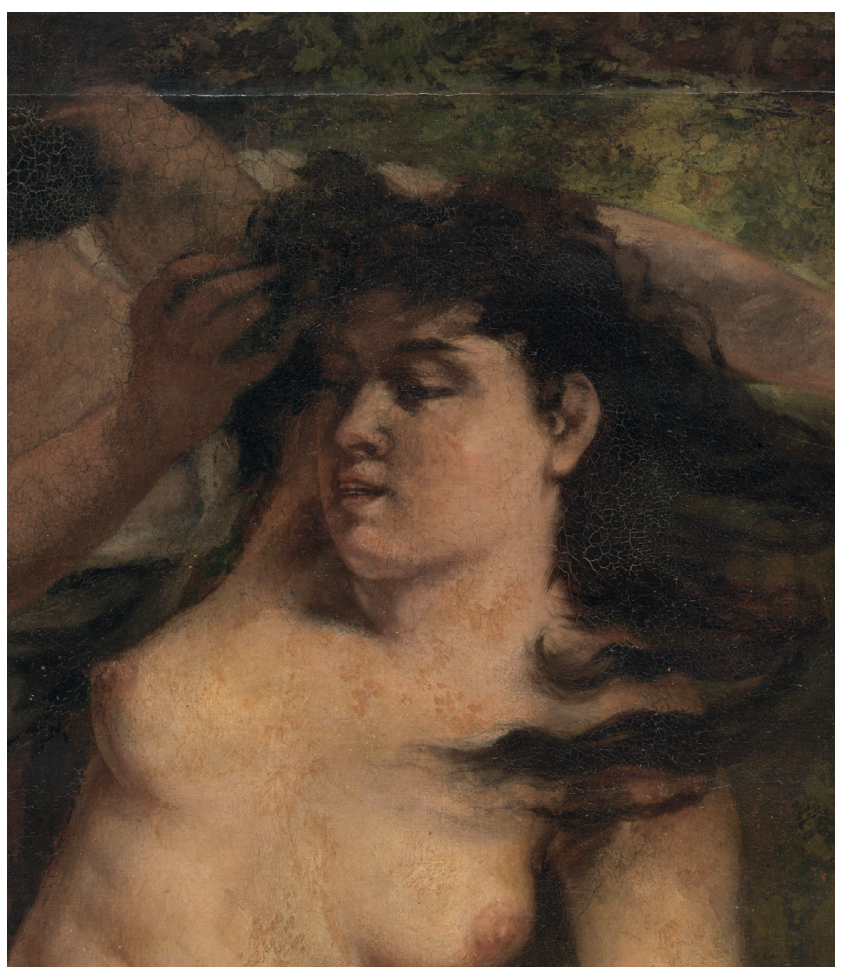

Fig. 5. Détail avant allègement avec les retouches désaccordées. (c) C2RMF/Jean-Louis Bellec. 
par un cadre doré, elle reste néanmoins une œuvre d'atelier expérimentale qui nous informe sur le processus créateur de Courbet.

$\mathrm{Au}$ Petit Palais, les archives en matière de restauration sont très lacunaires. L'usage était, jusqu'à une date récente, de ne conserver que des documents administratifs, essentiellement des devis et des factures. Fort heureusement, l'œuvre a été reproduite à plusieurs reprises depuis son entrée au musée. Ces clichés anciens ont été étudiés pour reconstituer l'évolution de son état. Leur archivage fait désormais partie de la documentation conservée précieusement dans le dossier de restauration.

Durant la restauration menée en 2014, l'enlèvement des repeints abusifs et l'allègement du vernis ont permis de retrouver une matière originale quasi intacte, très proche techniquement des séries de nus de la fin de la carrière de Courbet. L'œuvre, volée puis restituée au peintre, n'a jamais pu être achevée comme Courbet l'avait pourtant promis à Étienne Baudry qui souhaitait l'acquérir : «Veuillez, mon cher ami, croire que je suis toujours à votre disposition pour les terminer comme il était convenu en principe ${ }^{27}$. » L'historien de l'art peut se réjouir de cet empêchement qui a préservé ces Trois baigneuses à un stade intermédiaire d'achèvement, état que nous pouvons désormais observer dans sa vérité.

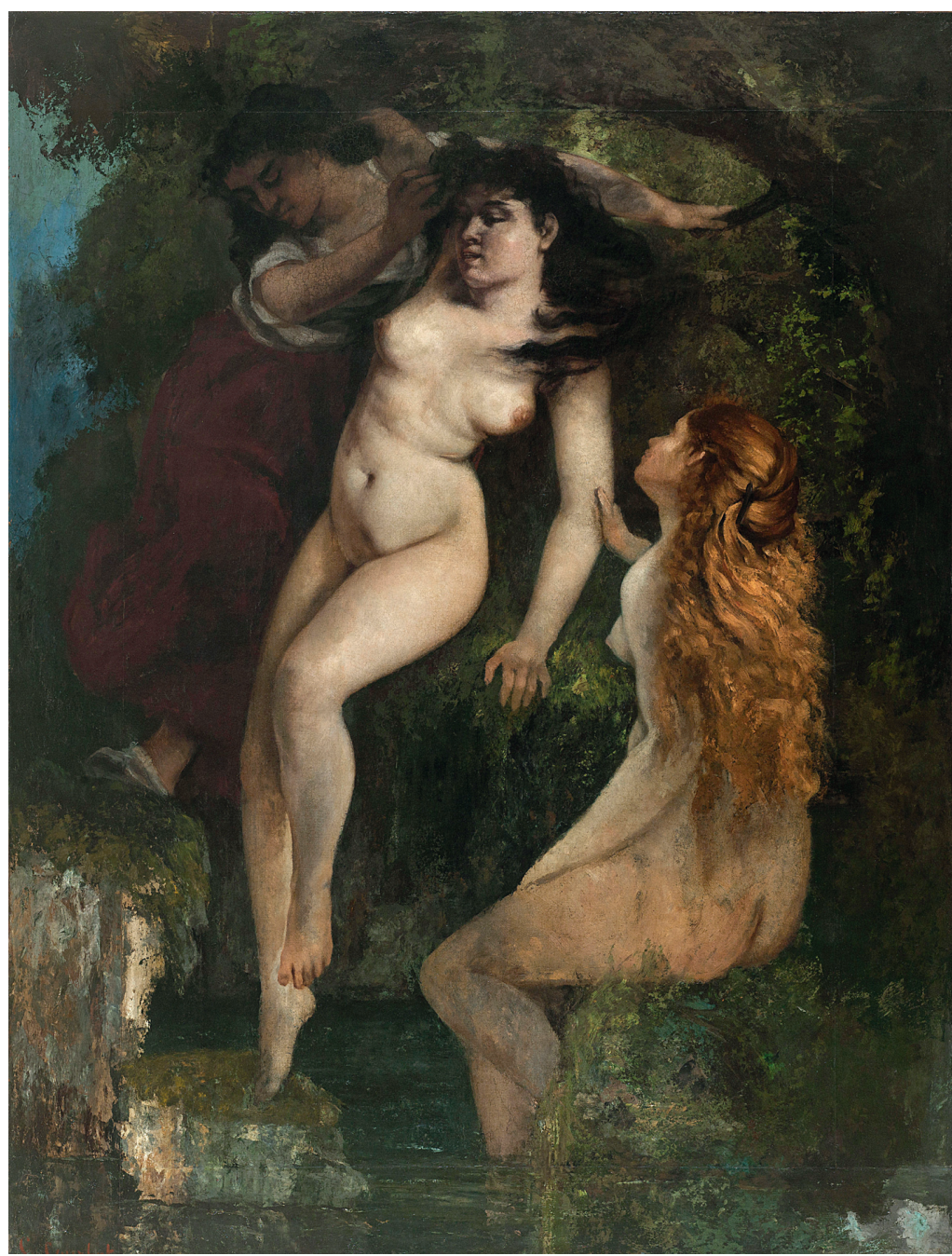

Fig. 6. Le tableau après restauration. (C Petit Palais/Roger-Viollet.
Notes

1. Lapauze, 1910, p. 79.

2. Chu, 1998, p. 140.

3. Gronkowski, 1929, p. 27.

4. Archives du Petit Palais, dossier d'œuvre PPP732.

5. Id. ibid.

6. Id. ibid.

7. Id. ibid. Facture du 20 octobre 1978, montant de l'intervention 850 francs.

8. Exposition Courbet und Deutschland, Hambourg, Kunsthalle du 19 octobre au 17 décembre 1978 et Städlische Galerie, Frankfurt du 17 janvier au 18 mars 1979 cat. $n^{\circ} 278$

9. Il s'agit probablement du cliché Bulloz encore disponible sur le site de la RMN Photo référence 05-521937.

10. Chu, 1996 et Bruyeron, 2011.

11. Bruyeron, 2011, p. 270.

12. Bruyeron, 2011, p. 274

13. Id. ibid. p. 262.

14. Labreuche, 2011, p. 288.

15. Archives du Petit Palais, dossier d'œuvre PPP732.
16. Bruyeron, 2011, p. 260.

17. Chu, 1996, p. 113

18. Bruyeron, 2011, p. 260.

19. Id. ibid., p. 270.

20. Id. ibid., p. 264.

21. Id. ibid., p.175.

22. Id. ibid., p. 262

23. Swicklick M., 1981.

24. Allègement du vernis réalisé avec un mélange Isopropanol/isooctane $(50 / 50)$.

25. En vieillissant l'indice de réfraction de l'huile se rapproche de celui de nombreux pigments, la matière devient alors plus transparente, les couches sous-jacentes sont plus visibles.

26. Vernis MS2A (résine cétonique réduite).

27. Chu, 1998, p. 464.

\section{Bibliographie}

Bruyeron R., 2011, Écrits, propos et témoignages, Hermann.

Chu P., 1996, Correspondance de Courbet, Flammarion.
Chu P., Zütter J., 1998, Courbet, artiste et promoteur de son cuvre, Flammarion.

Gronkowski, C. (préface), 1929, Exposition Gustave Courbet, Palais des Beaux-Arts de la Ville de Paris (Petit Palais) Bois-Colombes, Impr. des lettres et des Arts.

Labreuche P., 2011, Paris capitale de la toile à peindre, INHA/CTHS.

Lapauze H., 1910, Le Palais des Beaux-Arts de la Ville de Paris (Petit Palais), Imp. P. Renouard, Paris.

Léger C., 1929, Courbet, G. Grès.

Mottin B., 2007-2008, « Des œuvres à la genèse complexe, Courbet sous l'œil du laboratoire ", dans Exposition Gustave Courbet, Paris, Galeries nationales du Grand Palais ; New York, The Metropolitan Museum ; Montpellier, Musée Fabre, RMN, p.70-81.

Swicklick M., "French painting and the use of varnish, 1750-1900 ", Studies in the history of Art, Conservation research, National Gallery of Art, Washington, 1981. 